\title{
Assessment of Endometrial Thickness by TVS for Detection of Endometrial Carcinoma in Women with Post-Menopausal Bleeding
}

\author{
SABA AJMAL Dr. Syeda Khadija \\ University Institute of Radiological Sciences \& Medical Imaging Technology
}

\begin{abstract}
Objective: The aim of this study is to evaluate the role of endometrial thickness for detection of endometrial carcinoma in women with post-menopausal bleeding by use of Transvaginal ultrasound.

Method: In this systematic review database by Medline, PubMed, science direct and Obsgyne online library were queried for studies published between 2005 to 2020 by using specific MeSH terms. Abstract from original peerreviewed prospective Cohort studies that shows endometrial thickness with postmenopausal bleeding by specific ultrasound findings were included in this study.

Results: Clinical data were extracted from 14 studies in which approx. 2500 women included with postmenopausal bleeding and 215(8.6\%) cases were diagnose with endometrial carcinoma that shows endometrial thickness greater than $10 \mathrm{~mm}$ on Transvaginal ultrasound. The prevalence of endometrial cancer was high in patients who had endometrial thickness $>9.5 \mathrm{~mm}$. Women with PMB that diagnose with EC had ET was 10 to $16 \mathrm{~mm}$. Women with PMB less than $4 \mathrm{~mm}$ ET not diagnosed with EC.

Conclusion: In this reviewed study we concluded that women with PMB showing ET greater than $10 \mathrm{~mm}$ on Transvaginal ultrasound diagnosed with Endometrial carcinoma.

Keywords: Endometrial Thickness (ET), Endometrial carcinoma (EC), Post-menopausal bleeding (PMB), Transvaginal Ultrasound (TVS).

DOI: $10.7176 / \mathrm{JHMN} / 82-11$

Publication date: November $30^{\text {th }} 2020$

\section{Introduction}

Menopause is the termination of the reproductive phase of life in a woman. Menopause is a greek word and means 'men' (month) and pause (cessation) i.e. cessation of menstruation. ${ }^{1}$ The definition of PMB and what is clinically significant is however varied. By definition PMB is bleeding that occurs 12 months after the last normal period. However it is recommended that any vaginal bleeding that occurs 6 months after the last period ( presumed menopause ) should be investigated. ${ }^{2}$ About $10 \%$ of women with postmenopausal bleeding have a primary or secondary malignancy. Common malignancies among them are endometrial cancer $(80 \%)$, cervical cancer or an ovarian tumour.

Endometrial cancer is the second most common cancer associated with hereditary non-polyposis colorectal cancer. As postmenopausal bleeding is the commonest symptom of endometrial carcinoma, so patients presenting with it should be worked up on priority basis for early detection and management of endometrial carcinoma. ${ }^{3}$ In patients with postmenopausal bleeding, sonographic measurement of endometrial thickness is the first test to determine whether further investigations are needed to rule out malignancy. ${ }^{4}$ The initial assessment in all cases of PMB should be using the USG especially TVS. Measurement of the endometrium on USG should include the full double thickness of the endometrium with any content within the endometrial cavity. ${ }^{5}$

Transvaginal Sonography (TVS) is an efficient and acceptable non-invasive method for the early detection of endometrial pathology in postmenopausal women. The thickened endometrium during menopause is the most significant ultrasonographical criterion implicating its pathology. The role of TVS is well established in the search for endometrial hyperplasia and carcinoma. The results of various studies have shown that the TVS measurement of endometrial thickness is currently used as a diagnostic tool in patients with postmenopausal bleeding. The studies consistently show that an ultrasonographically measured endometrial thickness of 10 or $15 \mathrm{~mm}$ more chances of endometrial carcinoma as compare to those who had $4 \mathrm{~mm}$ to $5 \mathrm{~mm}$ or less almost completely excludes endometrial carcinoma. The advantage of TVS is that it can be performed with empty bladder and is convenient for the patient and at the same time, it is suitable for getting more correct gynecological diagnosis, especially in fatty women with a thick abdomen. ${ }^{6}$
\end{abstract}

\section{Method}

Search strategy

In this systematic review data extracted by Medline, PubMed, science direct and Obsgyne online library that were queried for studies published between 2005 to 2020 by using specific MeSH terms. 


\section{Study selection}

We extract data from 14 original peer-reviewed articles. In this reviewed article prospective Cohort studies that shows endometrial thickness with postmenopausal bleeding by specific ultrasound findings were included in this study. Endometrial thickness and the prevalence of endometrial (pre)malignancies were recorded.

\section{RESULTS}

In this systematic reviewed article we reviewed 14 articles that was published between 2005 to 2020 . A prospective Cohort study style was used for this reviewed article. 14 studied reporting on 2500 women with postmenopausal bleeding were included. Out of 2500 women $215(8.6 \%)$ women diagnosed with endometrial carcinoma. Those women who diagnosed with endometrial carcinoma all shows endometrial thickness greater than $10 \mathrm{~mm}$ on transvaginal ultrasound. The prevalence of endometrial cancer was high in patients who had endometrial thickness $>9.5 \mathrm{~mm}$. Women with PMB that diagnose with EC had ET was 10 to $16 \mathrm{~mm}$. Women with PMB less than $4 \mathrm{~mm}$ ET not diagnosed with EC. The result from this systematic reviewed article is that women with postmenopausal bleeding showing endometrial thickness greater than $10 \mathrm{~mm}$ on Tranvaginal ultrasound have a greater chance to diagnose with Endometrial carcinoma.

\section{Discussion}

Prof Ian Jacobs FRCOG Et al (2011) conducted a study on "Sensitivity of transvaginal ultrasound screening for endometrial cancer in postmenopausal women". It is case-control Cohort study of postmenopausal women who underwent TVS in the United Kingdom Collaborative Trial of Ovarian Cancer Screening following recruitment between April 17, 2001, and Sept 29, 2005..Our findings show that TVS screening for endometrial cancer has good sensitivity in postmenopausal women. The role of population screening for endometrial cancer remains uncertain, but our findings are of immediate value in the management of increased endometrial thickness in postmenopausal women undergoing pelvic scans for reasons other than vaginal bleeding.?

In 2008 CemilYaman Et al conducted a study on "The role of three-dimensional volume measurement in diagnosing endometrial cancer in patients with postmenopausal bleeding" Transvaginal ultrasound examinations was performed in 213 consecutive patients with a history of postmenopausal bleeding. The results of ultrasonography examinations were compared with the diagnoses on the basis of histologic examination. Minimal endometrial volume $(2.7 \mathrm{ml})$ and minimal endometrial thickness $(7 \mathrm{~mm})$ of endometrial carcinoma were defined as optimal cutoff values. In 42 patients, endometrial carcinoma was diagnosed. The area under curve of endometrial thickness was 0.85 (95\% CI 0.80-0.91). The comparison of the area under curve of receiver operating curves between endometrial volume and endometrial thickness revealed a significant difference $(p=$ 0.023).volume measurement by three-dimensional transvaginal ultrasound has a higher specificity, which means that it has the ability to better identify the negative cases compared to conventional ultrasound. Three dimensional transvaginal ultrasound is a helpful tool for diagnosing endometrial cancer in patients with postmenopausal bleeding. ${ }^{8}$

Cenk Yasa Et al (2016) conducted a study on "Evaluation of the diagnostic role of transvaginal ultrasound measurements of endometrial thickness to detect endometrial malignancy in asymptomatic postmenopausal women" A cohort study in a university hospital was undertaken with 276 consecutive asymptomatic postmenopausal women undergoing dilatation and curettage (D\&C) and hysteroscopy for an incidental finding of thickened endometrium ( $\geq 4 \mathrm{~mm}$ ) between 2003 and 2012. Different endometrial thickness cutoff values were tested on the basis of a pathologic report with carcinoma conditions (endometrial hyperplasia with atypia and endometrial carcinoma).With regard to carcinoma conditions, nine patients $(3.3 \%)$ had endometrial hyperplasia with atypia and eight patients $(2.9 \%)$ had endometrial carcinoma. Routine use of endometrial thickness measurement with TVUS does not seem to be an effective diagnostic tool for endometrial cancer because it has a low diagnostic performance in asymptomatic postmenopausal women. ${ }^{9}$

In 2015 Margit Dueholm Et al studied a topic "Diagnostic methods for fast-track identification of endometrial cancer in women with postmenopausal bleeding and endometrial thickness greater than 5 mm"This study used a prospective cohort of 174 women with postmenopausal bleeding and endometrial thickness of $5 \mathrm{~mm}$ or greater. As a first-line technique, pattern recognition on TVS, GIS correctly identifies 9 of 10 women with malignancy and is superior to pattern recognition on ES when insufficient samples are included. Endometrial pattern evaluated with TVS and GIS is a fast and efficient first-line diagnostic tool that outperforms ES in women with or without localized lesions. Malignant patterns on TVS/GIS should warrant fast-track evaluation, whereas women with benign patterns may be selected for office or operative hysteroscopy. ${ }^{10}$

In 2008 P. Tsikouras Et al conducted a study on "TV sonographic assessment in postmenopausal women with bleeding". This retrospective study was conducted to investigate the diagnostic prognostic value of only endometrial thickness in postmenopausal patients with postmenopausal bleeding. This study involved 275 postmenopausal women aged 47-81 years (median 62). Transvaginal sonography was performed in all women. About $89.2 \%$ of malignant diseases were discovered in the study women whose endometrial thickness was above 
$4 \mathrm{~mm}$. In postmenopausal symptomatic women premalignant or malignant causes of bleeding can not be excluded with just transvaginal ultrasound. ${ }^{11}$

In 2010 S.KAUR M Et al conducted a study on "Endovaginal Sonographic Evaluation of Postmenopausal Uterine Bleeding" The present study was conducted on postmenopausal women with postmenopausal bleeding. A total of 112 patients were observed from 2006 to 2008. A written informed consent was taken. EVS was done to measure endometrial thickness in post-menopausal women with postmenopausal bleeding (PMB).The mean endometrial thickness in PMW with PMB was $8.21 \pm 6.88 \mathrm{~mm}$ and in those without PMB was $3.83 \pm 2.14 \mathrm{~mm}$. With a cutoff value of $4 \mathrm{~mm}$ endometrial thickness, EVS showed a sensitivity of $100 \%$, specificity $73.33 \%$. EVS is a useful method for screening for endometrial abnormalities and we recommend its use in women with postmenopausal bleeding. Hence, unnecessary operations in postmenopausal women could be spared where the endometrium is ultrasonographically normal. ${ }^{12}$

In 2009 E. DREISLER Et al conducted a study on "Value of endometrial thickness measurement for diagnosing focal intrauterine pathology in women without abnormal uterine bleeding" A retrospective cohort study in which random selection from the Danish Civil Registration System was made: 1660 women aged 20-74 years were invited to participate and 686 women were eligible and accepted inclusion (429 pre- and257 postmenopausal). The women underwent TVS measurement of endometrial thickness and saline contrast sono hysterography (SCSH). In conclusion, the women without AUB, the TVS measurement of endometrial thickness was useful for exclusion of focal intrauterine pathology. However, when a thick endometrium is present, the diagnostic value of this measurement is limited. The 4-5-mm threshold used conventionally in symptomatic postmenopausal women is not transferable to women without AUB for excluding focal intrauterine lesion. ${ }^{13}$

In 2007 G. OPOLSKIENE Et al conducted a study on" Ultrasound assessment of endometrial morphology and vascularity to predict endometrial malignancy in women with postmenopausal bleeding and sonographic endometrial thickness $\geq 4.5 \mathrm{~mm}$ ". In This Cohort study 223 consecutive patients with PMB and sonographic endometrial thickness $\geq 4.5 \mathrm{~mm}, 120$ full-filled our inclusion criteria. They underwent transvaginalgray-scale and power Doppler ultrasound examination, which was videotaped for later analysis by two examiners with more than 15 years' experience in gynecological ultrasonography. Consecutive patients with postmenopausal bleeding,endometrial thickness $\geq 4.5 \mathrm{~mm}$ at transvaginal ultra-sound examination and without fluid in the intrauterine cavity underwent extended ultrasound examination as described below. The results of our study show that in a selected group of women with postmenopausal bleeding, endometrial thick-ness $\geq 4.5 \mathrm{~mm}$, good visibility of the endometrium and detectable Doppler signals in the endometrium, endometrial morphology as assessed by gray-scale ultrasonography and endometrial vessel characteristics as assessed by power Doppler ultrasonography are independently related to endometrial malignancy. Moreover, both add useful information to sonographic endometrial thickness as an indicator of the risk for endometrial carcinoma. ${ }^{14}$

In 2010 B Fan Et al worked on "Assessment of Transvaginal Sonographey Combined with Endometrial Cytology as a Mass Screening Method for Endometrial Cancer in Beijing". In phase one, 3308 women without known cancer were enrolled and TVS was performed on those with symptoms or endometrial cancer risk factors. Endometrial cytology was carried out on post-menopausal women with endometrial thickness $\geq 5 \mathrm{~mm}$ and on premenopausal women with endometrial thickness $\geq 10 \mathrm{~mm}$. The procedure of TVS involves a high frequency sensor, enabling deeper insertion into the vaginal path, consequently allowing scanning close to the uterus. This eliminates the need to fill the bladder before examination and allows the generation of clearer images than abdominal sonography. They used TVS to demonstrate that endometrial thickness is much greater in cancer or pre-cancer cases than in normal or benign cases in both pre- and postmenopausal women. This indicates that TVS examination of endometrial thickness is an effective method of endometrial cancer and pre-cancer screening Combined TVS and endometrial cytology resulted in $100 \%$ sensitivity and $99.0 \%$ specificity, reducing unnecessary D \& C by $91.7 \%$ and screening costs by $20.1 \%$. Combined TVS examination and endometrial cytology decreased potentially harmful examinations, patient suffering and medical costs, and is worth considering for broad implementation. ${ }^{15}$

MORTEZA Et al(2012) conducted a study on "COMPARING OF THE RESULTS OF TRANSVAGINAL ULTRASONOGRAPHY WITH DILATATION AND CURETTAGE FINDINGS IN EVALUATION OF ENDOMETRIAL CANCER IN WOMEN WITH POSTMENOPAUSAL BLEEDING" From April, 2008, through June, 2009, 98 women with PMB underwent TVS and histopathology evaluation of the endometrium after D\& C or after hysterectomy. Normal appearance at transvaginal sonographey was defined as a thin $(\leq 5 \mathrm{~mm})$ and diffuse smooth with smoothly marginated and homogeneously echogenic endometrium. However a suspicious appearance was defined as either irregular thickening of the endometrium or an inhomogeneous endoluminal mass or pedunculated endoluminal mass or any endometrial thickness above $5 \mathrm{~mm}$. At histologic examination: of 98 women, 44 (45\%) had an atrophic endometrium, 11 (11\%) had carcinoma, 10 (10\%) had hyperplasia, 28 (29\%) had polyps and $5(5 \%)$ were others (ovarian tumor, leiomyosarcoma and multiple or giant leiomyoma) For carcinoma, the sensitivity of TVS was $100 \%$, specificity was $41.38 \%$, PPV was $17.4 \%$, and NPV was $100 \%$.In addition, the specificity of TVS for atrophic endometrium was $100 \%$.TVS in patients with PMB is an excellent 
tool for the determination of whether further investigation with curettage or some form of endometrial biopsy is necessary and sensibly decreases the number of invasive methods. TVS also improves clinical decision- making, and the clinicians diagnostic certainty. ${ }^{16}$

Media Ghazi Sedeq Et al (2016) conducted a study "A prospective comparison of transvaginal, trans abdominal ultrasound and diagnostic curettage in the evaluation of endometrial pathology in Erbil" A prospective comparison study of transvaginal ultrasound, trans abdominal ultrasound and diagnostic curettage was conducted for evaluation of endometrial pathology in Maternity Teaching Hospital, Erbil city,Kurdistan region of Iraq, from September13th, 2013 to September14th, 2014. The study included 100 women presenting with abnormal uterine bleeding. Ultrasound findings were compared with histopathological results. Of 98 women $(2$ women were excluded from analysis), 19\% had atrophic endometrium and $67 \%$ had endometrial hyperplasia in histopathological finding. Transvaginal ultrasound sensitivity and specificity were $100 \%$ and $92.9 \%$, respectively while trans abdominal ultrasound sensitivity and specificity were $92.8 \%$ and $65 \%$, respectively. Transvaginal ultrasound scanning is an excellent tool for the determination of ET whether further investigation with histopathological examination of endometrial biopsy is necessary or not for women presented with abnormal uterine bleeding. ${ }^{17}$

A prospective study conducted by Ari Kim Et al (2015) on "Diagnostic utility of three-dimensional power Doppler ultrasound for postmenopausal bleeding" In this study we recruited 225 postmenopausal women with postmenopausal uterine bleeding who met the study criteria. Women who had hematologic disease, chronic medical diseases, or nonuterine pelvic diseases were excluded. Prior to endometrial biopsy, the patients underwent a baseline transvaginal ultrasound screening. Endometrial malignancy was confirmed in 28 cases (16.1\%), and endometrial hyperplasia was diagnosed in 17 cases $(9.8 \%)$. The prevalence of endometrial cancer was high in patients who had endometrial thickness $>9.5 \mathrm{~mm}(\mathrm{p}<0.001)$ and volume greater than $4.05 \mathrm{~mL}(\mathrm{p}<0.001)$. The diagnostic usefulness of 3D PD-US for endometrial diseases is promising in women with postmenopausal endometrial bleeding. ${ }^{18}$

\section{Conclusion:}

The conclusion we get from this systematic reviewed study shows that among women with postmenopausal bleeding endometrial thickness is substantially higher in women with endometrial carcinoma. So its concluded that if a women presenting with postmenopausal bleeding showing endometrial thickness greater than $10 \mathrm{~mm}$ on transvaginal ultrasound having a $90 \%$ chances to diagnose with Endometrial carcinoma. By using TVS we demonstrate that endometrial thickness is much greater in endometrial cases than in normal or benign cases in postmenopausal women. This indicates that TVS examination of endometrial thickness is an effective method for detection of Endometrial carcinoma.

\section{Recommendation}

EVS is a useful method for screening for endometrial abnormalities and we recommend its use in women with postmenopausal bleeding. The procedure of TVS involves a high frequency sensor, enabling deeper insertion into the vaginal path, consequently allowing scanning close to the uterus. This eliminates the need to fill the bladder before examination and allows the generation of clearer images than abdominal sonography. They used TVS to demonstrate that endometrial thickness is much greater in cancer or pre-cancer cases than in normal or benign cases in both pre- and postmenopausal women. Hence, unnecessary operations in postmenopausal women could be spared where the endometrium is ultrasonographically normal. Combined TVS examination and endometrial cytology decreased potentially harmful examinations, patient suffering and medical costs, and is worth considering for broad implementation.

\section{References}

${ }^{1}$ Kaur M* , Singh R**,et al;Endovaginal Sonographic Evaluation Of Postmenopausal Uterine Bleeding.

${ }^{2}$ https://indianmenopausesociety.org/wp-content/uploads/2018/12/Postmenopausal_bleeding.pdf

${ }^{3}$ Anon, Endometrial bleeding. Hum Report Update 2007; 13: 421-31.

${ }^{4}$ M. C. Breijer J. A. H. Pesters B. Capacity of endometrial thickness measurement to diagnose endometrial carcinoma in asymptomatic postmenopausal women obgyn.onlinelibrary.wiley.com/doi/full/10.1002/uog. 12306

${ }^{5}$ Kaur $\mathrm{M}^{*}$, Singh $\mathrm{R}^{* *}$, et al;Endovaginal Sonographic Evaluation Of Postmenopausal Uterine Bleeding Journal of Clinical and Diagnostic Research. 2010 April ;(4):2175-2182.

${ }^{6}$ Singh $\mathrm{R}^{* *}$,et al;Endovaginal Sonographic Evaluation Of Postmenopausal Uterine Bleeding (M.D), Asstt. Prof Adesh Institute Of Medical Sciences \& Research, Bathinda, Kaur 118/1 Gurjat (India). 144001. Ph.: 9779109999 , 981540999Kaur M

7 ProfIanJacobsFRCOGa,Aleksandra,Gentry-MaharajPhDa (2011).Sensitivity of transvaginal ultrasound screening for endometrial cancer in postmenopausal women. The Lancet Oncology, Volume 12, Issue( 1), 38-48. 
${ }^{8}$ CemilYaman ${ }^{\mathrm{a}}$ GernotTews ${ }^{\mathrm{a}}$ WernerPölz(2008) The role of three-dimensional volume measurement in diagnosing endometrial cancer in patients with postmenopausal bleeding, Gynecologic OncologyVolume 110, Issue 3, September 2008, Pages 390-395

${ }^{9}$ Yasa, C., Dural, O., Bastu, E. et al. Evaluation of the diagnostic role of transvaginal ultrasound measurements of endometrial thickness to detect endometrial malignancy in asymptomatic postmenopausal women. Arch Gynecol Obstet 294, 311-316 (2016). https://doi.org/10.1007/s00404-016-4054-5

${ }^{10}$ Dueholm M, Marinovskij E, Hansen ES, Møller C, Ørtoft G. Diagnostic methods for fast-track identification of endometrial cancer in women with postmenopausal bleeding and endometrial thickness greater than $5 \mathrm{~mm}$. Menopause. 2015 Jun;22(6):616-26. doi: 10.1097/GME.0000000000000358. PMID: 25535964.

${ }^{11}$ Tsikouras, P., Liberis, V., Galazios, G., Grapsas, X., Kantari, P., Papageorgiou, S., \& Maroulis, G. (2008). TV sonographic assessment in postmenopausal women with bleeding. European journal of gynaecological oncology, 29(1), 67.

12 (M.D), Asstt. Prof. Radiodiagnosis , ** (M.D), Asstt. Prof, *** (M.D), Asstt. Prof Adesh Institute Of Medical Sciences \& Research, Bathinda, (India). Corresponding Author: DR. Manjot Kaur 118/1 Gurjaipal Nagar, Jalandhar,(India). 144001. Ph: $9779109999,9815409999$.

${ }^{13}$ Dreisler, E., Sorensen, S. S., Ibsen, P. H., \& Lose, G. (2009). Value of endometrial thickness measurement for diagnosing focal intrauterine pathology in women without abnormal uterine bleeding. Ultrasound in Obstetrics and Gynecology, 33(3), 344-348.

${ }^{14}$ Opolskiene, G., Sladkevicius, P., \& Valentin, L. (2007). Ultrasound assessment of endometrial morphology and vascularity to predict endometrial malignancy in women with postmenopausal bleeding and sonographic endometrial thickness $\geq 4.5 \mathrm{~mm}$. Ultrasound in Obstetrics and Gynecology: The Official Journal of the International Society of Ultrasound in Obstetrics and Gynecology, 30(3), 332-340.

${ }^{15}$ Fan B, Zhao Q, Zhang S, Wang T, Deng X. Assessment of Transvaginal Sonography Combined with Endometrial Cytology as a Mass Screening Method for Endometrial Cancer in Beijing. Journal of International Medical Research. June 2010:803-809

16 TAHMASEBI MORTEZA*,IMEN MARYAM,RAZI TAGHI COMPARING OF THE RESULTS OF TRANSVAGINAL ULTRASONOGRAPHY WITH DILATATION AND CURETTAGE FINDINGS IN EVALUATION OF ENDOMETRIAL CANCER IN WOMEN WITH POSTMENOPAUSAL BLEEDING JUNDISHAPUR SCIENTIFIC MEDICAL JOURNAL 2012, Volume 11, Number 3 (78); Page(s) 229 To 240. 17 A prospective comparison of transvaginal, 1, transabdominal ultrasound and diagnostic curettage in the evaluation of endometrial pathology in Erbil Zanco J. Med. Sci., Vol. 20, No. (1), 2016 http://dx.doi.org/10.15218/zjms.2016.0010

${ }_{18}$ Diagnostic utility of three-dimensional power Doppler ultrasound for postmenopausal bleeding, Ari Kim,Ji Young Lee,Sungwook Chun,Heung Yeol Kim,Taiwanese Journal of Obstetrics and Gynecology 